\title{
Multicasting optical interconnects using liquid crystal over silicon devices
}

\author{
Andreas Georgiou, ${ }^{1, *}$ Jeroen Beeckman, ${ }^{2}$ and Kristiaan Neyts ${ }^{2}$ \\ ${ }^{1}$ Photonics and Sensors Group, Electrical Engineering Division, Department of Engineering, \\ University of Cambridge, Cambridge CB3 OFA, UK \\ ${ }^{2}$ Department of Electronics and Information Systems, Ghent University, Sint-Pietersnieuwstraat 419000 Gent, Belgium \\ ${ }^{*}$ Corresponding author: ag245@cantab.net
}

Received June 7, 2010; revised December 18, 2010; accepted December 20, 2010; posted December 23, 2010 (Doc. ID 129654); published February 18, 2011

\begin{abstract}
This work presents the characteristics and expected capabilities of an optical interconnect that uses a diffractive liquid crystal over silicon (LCOS) device as a routing element. Such an interconnect may be used in a neighborhood's optical network to distribute high definition television, thus avoiding an electronic or optical transmitter for each user. The optimal characteristics of the LCOS device are calculated in terms of pixel number and silicon area and found to be feasible with today's technology. Finally, its performance in terms of optical efficiency and number of output ports is evaluated and found suitable for a neighborhood with hundreds of households. (c) 2011 Optical Society of America
\end{abstract}

OCIS codes: $\quad 050.1970,060.6718,070.6120,090.1995$.

\section{INTRODUCTION}

Future optical networks will rely more on optical interconnects. They could be used to restore a network after a link failure, dynamically distribute bandwidth, and remotely connect or disconnect users. When fibers to the home (FTTH) are more widely installed, optical interconnects will provide a flexible and low-cost method for adding or removing high bandwidth users into the network; for example, in video signal distribution where a large number of outputs and multicasting is required.

The download rate of any home user will be significantly larger than the upload rate. Therefore, the strain in the network will be from the service provider to the user. A television viewer may easily be subscribed to a couple of hundred channels to which he/she wants instant access. If these channels are in $1080 \mathrm{p}$, possibly some with three-dimensional functionality, the bandwidth requirements are significant. In addition, if some users request video on demand, the total number of video channels being delivered in a neighborhood from the service provider could reach a thousand. Personalized content will be encrypted (similar to a wireless network). With a thousand channels at high definition, the total bandwidth requirement is of the order of gigabits per second. At this bit rate, the conversion from optical to electrical signal is expensive and must be made at the user. Routing the signal in the optical domain eliminates the need for an optical-electricaloptical conversion at the exchange and a high-speed electronic router.

Figure 1 shows how the switch could be used in a HDTV distribution network. By generating a high-power optical signal and then distributing, using an optical interconnect, the use of an electronic or optical transmitter for each user is avoided, reducing hardware and installation costs. In the future, a single powerful laser (of which its polarization is carefully controlled) could provide power to tens or hundreds of users and be distributed by an optical interconnect.
Note that liquid crystal materials, due to their rod-shaped molecular structure, will affect each polarization of the laser in a different way. This is not an issue here because the laser source will be physically next to the LCOS device and its polarization will be controlled. By having the polarization parallel to the liquid crystal molecules, the polarization effects are controlled and the phase excursion of the incident wave is maximized. Some small polarization modulation may be observed between pixels of different voltage [1] but their effect will be significantly smaller than the fly back effects (discussed in Subsection 3.D.3).

The advantage of holographic interconnects over competing technologies is their ability to only route power to the selected ports. Thus efficiency is not affected by the number of potential users, $S_{\max }$, but by the number of connected users, $S$. In a holographic switch, the power per output channel is given by $\eta P_{\text {in }} / S$ (see Fig. 1 ) where $\eta$ is the power efficiency of the interconnect and $P_{\text {in }}$ is the power input into the switch. This makes holographic switches ideal when the number of potential users, $S_{\max }$, is large but at any moment only a fraction of those are connected, like the distribution of video in a neighborhood. Service providers like to have all households as potential customers, $S_{\max }$, but, at any moment, only a number, $S$, of them is connected.

Other technologies can multicast, but they base their operation in blocking light from the nonconnected users. This brings the power per user down to $\eta P_{\text {in }} / S_{\max }$ compared to $\eta P_{\text {in }} / S$ for a holographic switch. Thus a holographic switch gives the flexibility to have a large number of potential users, $S_{\text {max }}$, while not losing any power for this flexibility.

Holographic interconnects can rewrite the phase profile of the beam, and in doing so can correct for defocus, astigmatism, or misalignment, thus improving the power coupling into the output fibers.

The development of holographic interconnects started more than a decade ago and over this time has undergone 


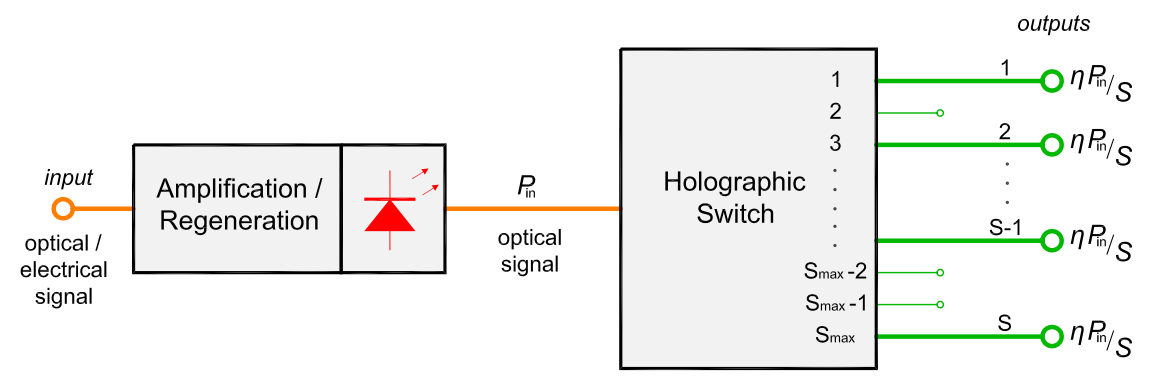

Fig. 1. (Color online) Position of a holographic switch in an optical network.

slow but steady development. The first holographic switch demonstrated used a binary ferroelectric device with optical loss of $16.9 \mathrm{~dB}$ [2-4]. Since then, the LCOS technology has evolved significantly. There was a shift in favor of nematic LCOS devices with analog phase excursion. At the same time, lithographic techniques have improved and now there are LCOS devices with deposited dielectric mirrors that are effectively $100 \%$ reflective. Holographic switches as a part of reconfigurable optical add-drop multiplexer have also been recently demonstrated [5].

This paper starts by presenting the proposed optical arrangement for the system together with theory on holographic switching. Section 3 presents the sources of loss in a holographic interconnect and gives a theoretical estimate of the system efficiency. Section $\underline{4}$ gives an estimate to the number of output ports possible in a holographic switch. Section 5 gives an overall presentation of the proposed system, suggesting characteristics of the LCOS device. Finally, the conclusions are given.

\section{HOLOGRAPHIC INTERCONNECTS}

Holographic optical interconnects use diffraction to route light to the target output fibers. Figure 2 shows the optical arrangement used in past demonstrations of holographic interconnects [6]. The beam emerging from the input fiber, with a Gaussian-like profile, expands and then it is collimated by a positive lens. The LCOS device, with dimensions $L \times L$, modulates the phase of the incident beam, introducing highfrequency components. The reflected beam is focused by the lens that, in effect, Fourier transforms the beam profile. This makes the beam focus move on a different position or positions depending on the phase pattern on the device.

The illumination profile on the device can be approximated by a Gaussian profile. Its width is given by the Fourier trans- form of the near field that is also a Gaussian. The two beam widths are related by

$$
2 w_{d}=\frac{4 \lambda}{\pi} \frac{f}{2 w_{i}},
$$

where $f$ is the focal distance of the lens, $\lambda$ is the optical wavelength, $2 w_{d}$ is the beam width on the LCOS device, and $2 w_{i}$ is the beam width of the input source (see Fig. 2). In a Gaussian profile, the beam width is defined as the circle diameter where intensity drops to $1 / e^{2}$ of its peak value.

The size of the output beam is also given by Eq. (1). If the beam is not heavily apodized, which must be the case to minimize optical losses, $2 w_{o}$, the output beam width is equal to $2 w_{i}$, the input beam width. If the input fiber has the same diameter as the output fibers, the output beam would fit exactly into the output fiber. Increasing the diameter of the output fiber relaxes the tight constraints on spot size and positioning, and coupling efficiency will improve. This could be done by the use of a multimode fiber (MMF), a taper fiber [7], or a microlens. The optical fibers are expected to operate at $1.3 \mu \mathrm{m}$, $1.5 \mu \mathrm{m}$, or $850 \mathrm{~nm}$ and be arranged in a rectangular grid, as shown in Fig. 3. The input fiber will be placed in the center of the grid. The LCOS device may have a small tilt in relation to the input fiber to eliminate any unwanted reflections entering back into the input fiber. As the cladding of single-mode fiber (SMF) has a $125 \mu \mathrm{m}$ diameter, the spacing between output fiber cores must also be at least $125 \mu \mathrm{m}$.

\section{EFFICIENCY}

The optical efficiency of an interconnect is defined as the power of the output signal over the input signal. Each user must receive a certain minimum power. Given that input power $P_{\text {in }}$ cannot be controlled by the interconnect, efficiency
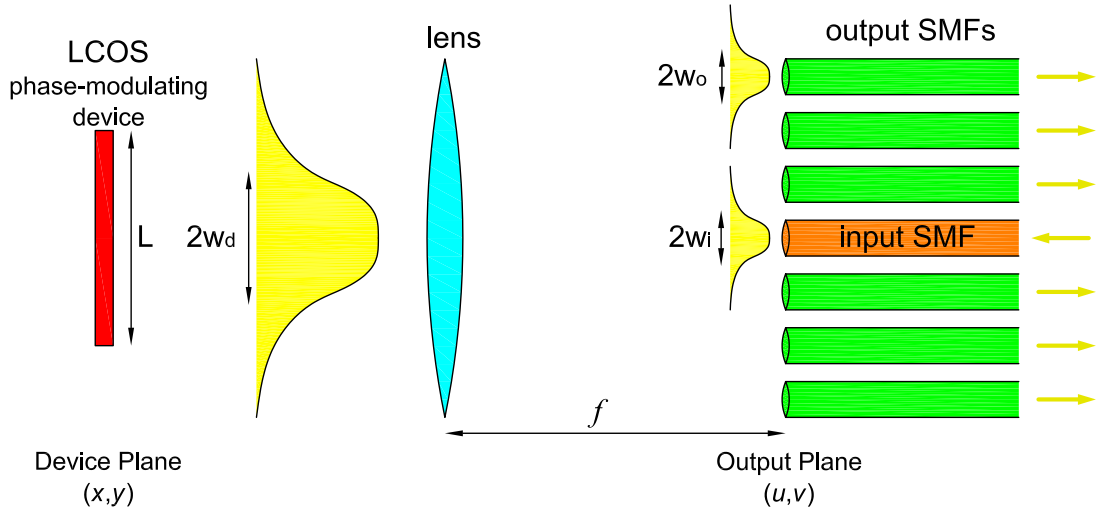

Fig. 2. (Color online) Optical arrangement of a holographic interconnect. 


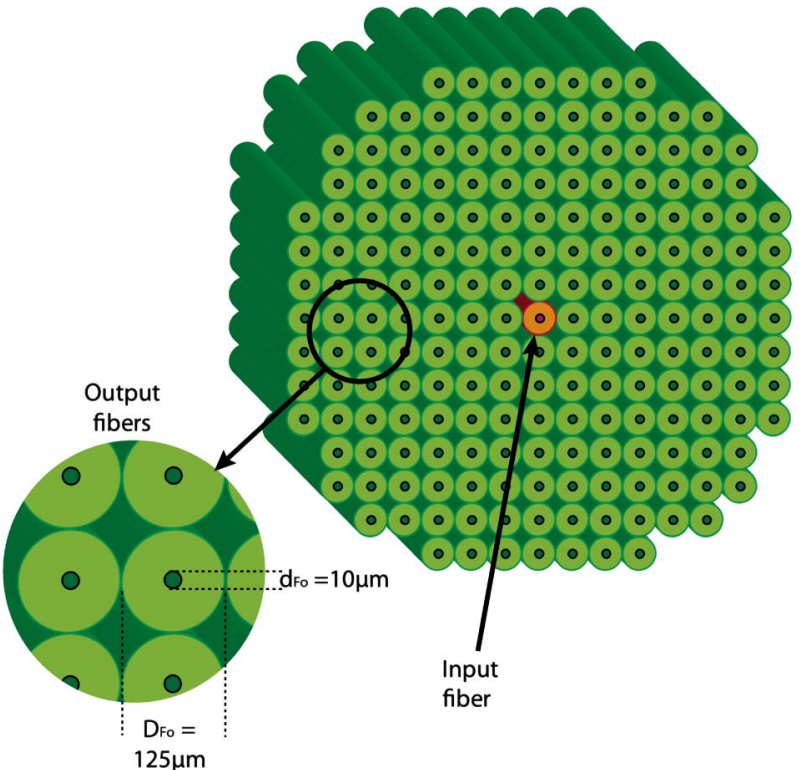

Fig. 3. (Color online) Suggested arrangement of the input and output fibers (here $S_{\max }=192$ ).

$\eta$ determines the maximum number of connected users. Thus, high efficiency will allow more users to be connected.

Values of acceptable efficiency vary widely and depend on the application and other functions of the system. MEMS with efficiencies better than $-7 \mathrm{~dB}$ [8] have been reported with up to 256 inputs and outputs. However, the particular system has a different role in the network as it cannot multicast or broadcast. Because holographic switches can dynamically control the power of each output channel, outputs connected with lossy links may be boosted to achieve fairer power distribution, and this will allow more users to be connected.

Efficiency strongly depends on the choice of the appropriate LCOS device. Most commercial LCOS devices are designed for display applications where pixel count is maximized and device area is minimized. Some LCOS devices have been designed specifically for infrared operation, like the Roses chip [2-4]. In this work, four custom devices are proposed and their performance is evaluated. The first device, refered to as the LCOS for optical infrared switching (LOIS), is larger in terms of active area and has fewer pixels compared to most commercial devices. Scaled down versions of LOIS, the $m \mathrm{LOIS}$, the $\mu \mathrm{LOIS}$, and the $n \mathrm{LOIS}$, are also considered. In addition, a number of commercial devices are presented and are shown together with the proposed ones in Table $\underline{1}$ (note that for commercial devices, $N$ and $L$ refer to the vertical direction).

The optical loss not directly linked with the holographic nature of the interconnect is not considered in this work. These include back reflections, mirror reflectivity, and coupling losses. Note that the efficiency of the switch could be significantly affected if the optical system and the fiber array are not designed appropriately.

Although the hologram can deflect the beam by a range of angles, the Fourier lens must be diffraction limited for all the range. In addition, the launch angle into the output fibers should be normal to maximize insertion efficiency. Aspheric lenses with these characteristics are now available. Referred to as telecentric $F$-theta lenses [9], they are widely used as scan lenses in optical coherence tomography and confocal microscopy. They will ensure that the beam incidents on the output fiber array at normal angle (telecentric operation) and that the position of the focus is proportional to the field angle ( $F$-theta operation). In addition, the focal plane is flat instead of curved. This is shown graphically in Fig. $\underline{4}$. An alternative way to eliminate the issue of the oblique incidence is to use a second hologram that deflects the beam in the opposite direction by the same amount. This arrangement, usually referred to as router-selector architecture, is used to transform the switch into a crossbar switch [10].

Another challenge is the construction of the densely packed array shown in Fig. 3. Such an array is feasible but it is likely to be an engineering challenge. Nevertheless, waveguide technology has been constantly improving. Waveguides with 50,000 fibers are commercially available (e.g., Sumitomo Image Guide IGN-20/50) and are used for optical fiber image guides with core spacing of less than $10 \mu \mathrm{m}$. Fibers, and especially fiber arrays, will have their core slightly misplaced and, if this is not considered, efficiency will be further reduced. There are a few ways to minimize and even eliminate the issue. The hologram can be adapted by using feedback (such as signal intensity) from the user to reconfigure for the revised positions. This must include some kind of feedback from the fiber. Another way to eliminate this issue is to increase the diameter of the SMF using a taper fiber [7] or a microlens. This technique has the advantage that the effective diameter of the core increases while the fiber remains single mode. Finally, a MMF may be used, although it may not always be desirable to use both SMFs and MMFs in the same network. Nevertheless, for short distances, the MMF may be the most cost-effective solution in terms of hardware.

Table 1. Characteristics of the Commercial LCOS Chip Together with LOIS, the Proposed LCOS Chip

\begin{tabular}{|c|c|c|c|c|c|c|c|c|}
\hline Device Name & $\Delta / \mu \mathrm{m}$ & $g / \mu \mathrm{m}$ & $N$ & $F$ & $L / \mathrm{mm}$ & Area $/ \mathrm{mm}^{2}$ & Dimensions & Manufacturer \\
\hline LOIS & 18.0 & 0.25 & 1024 & 0.97 & 18.4 & 339 & $18.4 \times 18.4$ & - \\
\hline$m$ LOIS & 18.0 & 0.25 & 720 & 0.97 & 13.1 & 169 & $13.2 \times 13.2$ & - \\
\hline$\mu$ LOIS & 18.0 & 0.25 & 512 & 0.97 & 9.2 & 85 & $9.1 \times 9.1$ & - \\
\hline$n$ LOIS & 18.0 & 0.25 & 164 & 0.97 & 2.9 & 8.7 & $2.9 \times 2.9$ & - \\
\hline 4 K2 K D-ILA & 6.8 & 0.25 & 2400 & 0.93 & 16.3 & 364 & $16.3 \times 26.1$ & JVC \\
\hline BR1920HC & 9.5 & 0.40 & 1200 & 0.92 & 11.4 & 208 & $11.4 \times 18.2$ & Brillian [23] \\
\hline JVC Professional & 9.5 & 0.45 & 1080 & 0.91 & 10.3 & 187 & $10.3 \times 18.2$ & JVC \\
\hline Qualia & 9.0 & 0.35 & 1080 & 0.92 & 9.7 & 168 & $9.7 \times 17.3$ & Sony \\
\hline JVC Consumer & 8.1 & 0.45 & 1080 & 0.89 & 8.7 & 136 & $8.7 \times 15.6$ & JVC \\
\hline Sony XBR & 7.0 & 0.35 & 1080 & 0.90 & 7.6 & 102 & $7.6 \times 13.4$ & Sony \\
\hline
\end{tabular}




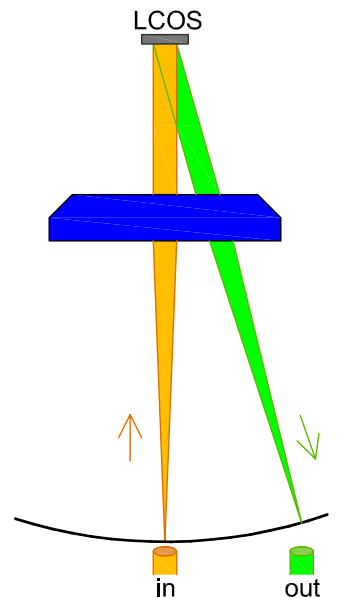

Fourier lens with curved focal plane

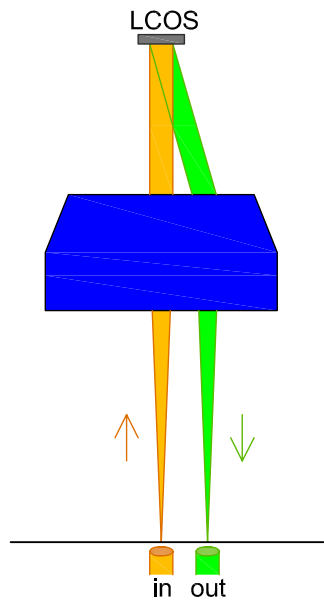

telecentric f-Theta Fourie lens with flat focal plane
Fig. 4. (Color online) Telecentric $F$-theta lens will focus the beam at normal angle to the focal plane, its position will be proportional to the input field angle, and the focal plane is flat.

The losses considered in this work are associated with the (i) device fill factor (ii) beam apodization (iii) hologram efficiency, and (iv) phase rendering. In the following four subsections, these losses are considered in detail.

\section{A. Apodization}

Apodization losses refer to the trimming of the Gaussian profile by the LCOS device. The amount of apodization is determined by the focal distance of the lens: a large focal distance will create a broad Gaussian function with large trimming of the profile. There is a trade-off in choosing the optimal focal distance. It is desirable to minimize losses by having small $f$ and concentrating more power on the device. At the same time, it is also desirable to use all the available pixels because this increases the number of output ports. Given the importance in optical efficiency, it may be appropriate to use more silicon area rather than increase loss. Apodization also changes the size and the shape of the focal points, reducing power coupling into the output fibers. The amount of energy landing on the active area of the device, $P_{d}$, is given by

$$
P_{d}=\frac{1}{w_{d} \sqrt{2 \pi}} \int_{-L / 2}^{x=+L / 2} \int_{-L / 2}^{y=+L / 2} e^{-\left(x^{2}+y^{2}\right) / 2 w_{d}^{2}} \mathrm{~d} x \mathrm{~d} y,
$$

where $w_{d}$ is a function of focal distance, $f$. The apodization loss for different focal distances was calculated by integrating the power over the square area of the device using the above integral. Figure $\underline{5}$ shows this variation with the horizontal axis showing the beam size in comparison to the device size and the vertical axis showing the optical loss. It can be seen that, for a beam width of about $0.4 L$, the apodization is very small and less than $-0.1 \mathrm{~dB}$. Above this, apodization loss is significant. Thus, it is recommended that the beam width is about $0.4 L$

\section{B. Fill Factor}

LCOS devices are silicon devices in which a layer of reflective metal, like aluminum, is deposited on top of the silicon backplane. Pixels act both as mirrors and electrodes; thus, they must be separated by a non-conductive area, refered to as in-

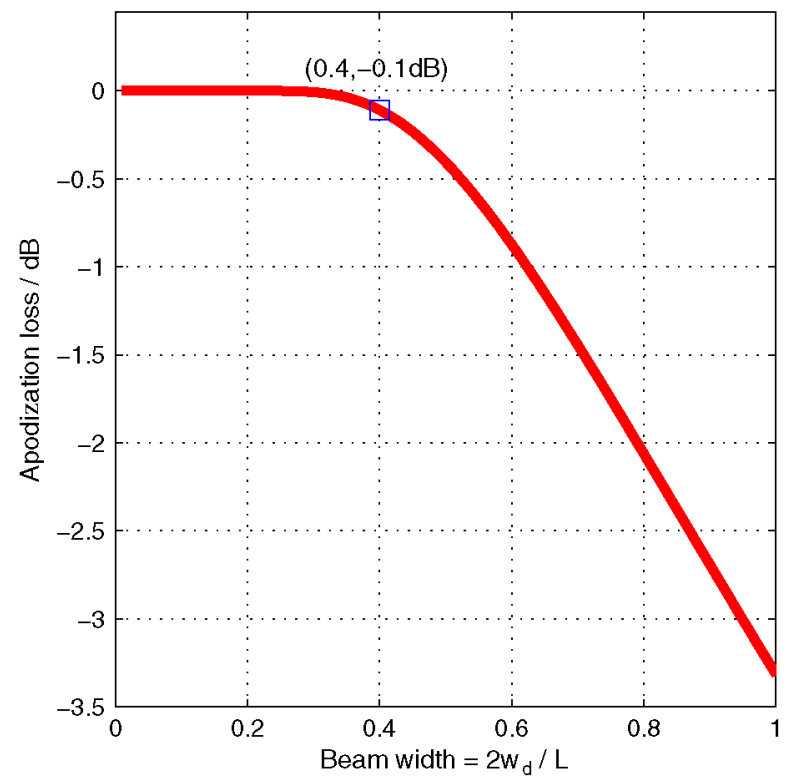

Fig. 5. (Color online) Apodization losses as a function of the beam width.

terpixel gap or dead space. In commercial devices, this space can be as low as $0.25 \mu \mathrm{m}$ [11]. Note that devices with dielectric mirrors may have zero interpixel gap, but the associated fringing fields are significant. Interpixel gap reduces the average reflectivity of the device by a factor $F$, the fill factor, which is equal to

$$
F=\left(\frac{\Delta-g}{\Delta}\right)^{2},
$$

where $\Delta$ is the pixel pitch in the $x$ and $y$ directions on the device plane and $g$ is the interpixel gap as shown in Fig. 6 . Interpixel gap and pixel pitch are the same in both directions.

For a rectangular device with active area $L \times L$ and $N \times N$ pixels, the fill factor can also be expressed as

$$
F=\left(1-\frac{g}{L} N\right)^{2}
$$

In this equation, the interpixel gap, $g$, is fixed by the lithographic process. The dimensions of the active area, $L$, heavily affects the cost of the device and thus it is also constrained. Only the number of pixels, $N$, may be treated as a free variable when optimizing the system. Increasing the number of pixels for a fixed silicon area will increase the number of the output fibers (smaller pixels thus larger deflection angle), but will also increase the fill factor losses. Thus, for a given number of outputs, the minimum number of pixels must be used.

Despite the large cost of silicon per square millimeter, an interconnect will have a higher value and longer lifetime than a consumer appliance, allowing larger silicon devices. Increasing the size improves the fill factor and the overall efficiency of the system. It is proposed that LOIS has only 1024 pixels but an active area of $18.4 \mathrm{~mm} \times 18.4 \mathrm{~mm}$, thus having a fill factor loss of only $0.15 \mathrm{~dB}$.

\section{Hologram Efficiency}

Hologram efficiency refers to the theoretical maximum energy a phase-only hologram can deliver to the target positions. The 


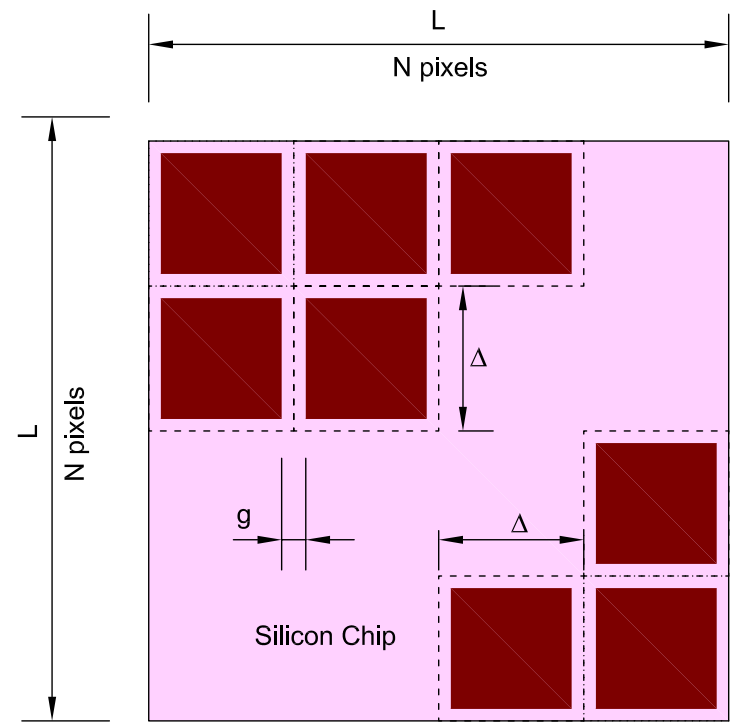

Fig. 6. (Color online) Dimensions of an LCOS device designed for holographic optical interconnects.

hologram can modulate only the phase of the incident beam and not its amplitude. This introduces ghost orders that reduce the diffraction efficiency. Depending on the arrangement of the output ports, the diffraction efficiency of the hologram may vary from $0 \mathrm{~dB}$ (for a blazed grating) to about $-1 \mathrm{~dB}$ (see Fig. 7).

There is not an analytical way to calculate the exact hologram diffraction efficiency for any arbitrary port arrangement. However, the worst case scenario is for two output ports when the diffraction loss can be analytically calculated and it is equal to $2 \operatorname{sinc}\left(\frac{\pi}{2}\right)$ or $-0.9 \mathrm{~dB}$ (this is found in the same way as the efficiency of a binary-phase hologram, see Subsection 3.D.2). Increasing the number of multicasting outputs reduces the power per output fiber, $\eta P_{\text {in }} / S$, but improves the overall efficiency, $\eta$. The best-case scenario is when there is only one output, in which case the diffraction efficiency is unity.

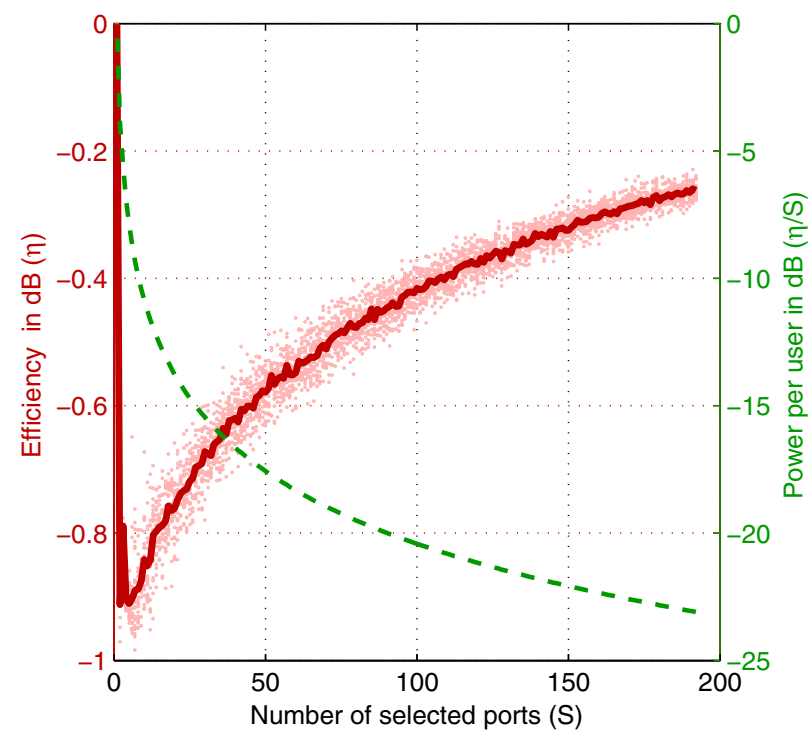

Fig. 7. (Color online) Hologram diffraction efficiency versus number of output users. Efficiency increases, thus making holographic interconnects best suited for networks with many users.
In order to estimate the ideal diffraction efficiency of the system, a number of holograms were computed using the output plane phase optimization [12] method and direct binary search $[\underline{13}, \underline{14}]$. The number of selected output ports, $S$, was varied from one to 192 . For each $S, 40$ different combinations of output fibers were made and, for each combination, a hologram was designed to route light to them. The output fibers were placed on a regular grid of $15 \times 15$ with the central fiber of the grid being the input, as shown in Fig. 3. Eight fibers on each corner were not used in order to form a more circular arrangement. Thus, the total number of output fibers was 192. The solid curve in Fig. 7 shows the mean diffraction efficiency for any number of spots from one to 192 . The dots show diffraction efficiencies of individual holograms. It can be seen that the optical loss is always better than $-1 \mathrm{~dB}$ and improves as the number of output channels increases. The dashed curve shows the power per user. The power per user is not constant but increases as the number of users decreases, thus making better utilization of the available power.

Note that, if necessary, the hologram design can reduce the crosstalk of the interconnect down to acceptable levels. This can be done by changing the hologram pattern in a way that cancels the effect of device imperfections [15]. Many authors have investigated ways to calculate in real time these errors and compensate for them [16]. Finally, the great strength of holograms lies in the fact that most device imperfections will be transformed into the Fourier domain; thus, they are likely to arrive either on the zero order or as high-frequency component noise. Currently, one-to-one optical switches are used as commercial systems and their performance in terms of crosstalk is acceptable [5].

\section{Phase-Rendering Losses}

Real LCOS devices do not render the phase profile perfectly and, thus, additional optical losses are introduced. There are three main sources of phase errors in a device: spatial quantization or pixelation [17] , phase quantization [2,ㅡㄹ , and electric field fringing $[\underline{18}, \underline{19}]$.

\section{Spatial Quantization}

Square pixels on an LCOS device act as apertures forming a far field on the output plane [17]. All pixels have the same shape but are shifted in space. Space shifting on the hologram plane translates to phase shifting in the output plane. Therefore, on the output plane, the far field of each pixel will have the same amplitude and position but different phase. Adding the effect of all pixels together, it will form a far-field amplitude envelope with the same shape as the far field of a single pixel. The far field of a square pixel is a two-dimensional sinc function and it is given by

$$
\eta_{\text {sinc }}=F \operatorname{sinc}^{2}(u K) \operatorname{sinc}^{2}(v K),
$$

where $K$ is given by

$$
K=\frac{\pi \Delta}{\Delta-g},
$$

and $u$ and $v$ are the normalized horizontal and vertical coordinates on the output plane. The normalized coordinates of 
the output plane are unity at position $\frac{\lambda}{\Delta} f$ or at deflection angle $\frac{\lambda}{\Delta}$.

Figure 8 shows the sinc envelope in one dimension when the fill factor is unity (solid curve). The thick curve shows the range of attenuation with the commercial devices shown in Table 1, and the dashed curve is for the four LOIS devices. The horizontal line shows the normalized deflection angle. The maximum normalized deflection angle in a holographic interconnect is \pm 0.5 when the period is two pixels. Above that spatial frequency, aliasing occurs.

In two dimensions, the sinc envelope will form a top-hat function. The nearer to the center a beam is deflected, the less the attenuation. Because the area available on the output plane is limited, the more ports that are placed, the more attenuation must be sustained by the ports further away from the zero order. This is shown on a two-dimensional contour map in Fig. 9. It corresponds to the ideal case when the fill factor is unity. It shows that, if a maximum $-0.5 \mathrm{~dB}$ loss is acceptable due to the sinc envelope, only the area of the central contour may be used. This corresponds to a fraction of 0.15 of the total output plane area. The ratio of the area where output ports may be placed over the total area will be denoted by the parameter $\alpha$. If higher loss is acceptable, $\alpha$ is larger. For an acceptable loss of $-3 \mathrm{~dB}, \alpha$ is about 0.6 and this increases the area of the useful output plane and the number of the output ports. Loss for real devices will be lower due to the reduced fill factor. The amount of available area for a given efficiency reduction due to the sinc envelope is given in Fig. 10.

\section{Phase Quantization}

Phase quantization is caused by the limited palette of voltages the silicon backplane can provide. In general, a digital-toanalog converter (DAC) will provide the analog voltage driving the liquid crystal cell. More phase levels will increase the complexity of the DAC and could reduce its speed. Therefore, it is important to design a chip with the least possible number of phase levels.

For a general multicasting hologram, the efficiency reduction due to phase quantization cannot be calculated analyti-

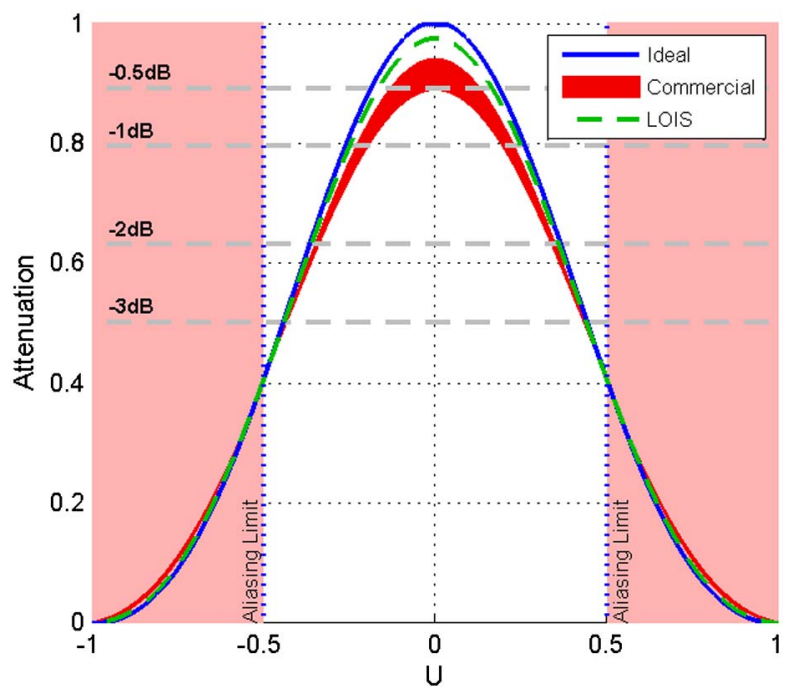

Fig. 8. (Color online) Sinc envelope formed at the output plane due to the square pixel shape. Keeping fibers near the center reduces attenuation.

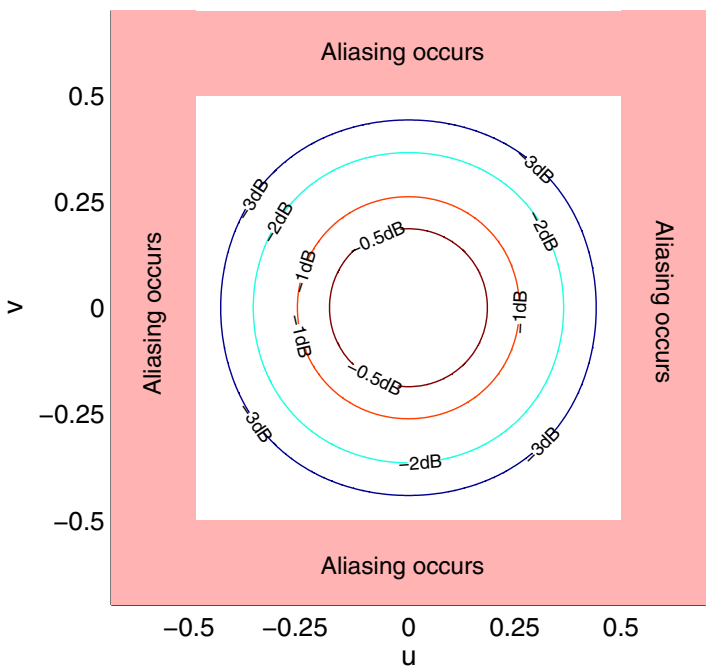

Fig. 9. (Color online) Two-dimensional sinc envelope attenuation as a contour map.

cally. However, the efficiency $\eta_{\mathrm{pq}}$ of a phase-quantized blazed grating can be calculated, and it is equal to

$$
\begin{gathered}
\eta_{\mathrm{pq}}=\left[\int_{-\pi / p}^{\theta=\pi / p} \cos (\theta) \mathrm{d} \theta\right] /\left[\int_{-\pi / p}^{\theta=\pi / p} 1 \mathrm{~d} \theta\right] \\
=\operatorname{sinc}\left(\frac{\pi}{p}\right)
\end{gathered}
$$

where $p$ is the number of available phase levels and $\theta$ corresponds to the phase delay introduced by a pixel relative to the phase of the target spot. The first square parenthesis gives the intensity of the beam when there were only $p$ phase levels and the second square parenthesis when there are infinite phase levels. Efficiency is the ratio of the two.

The effect of phase quantization for holograms with more than one output was estimated by computing holograms with

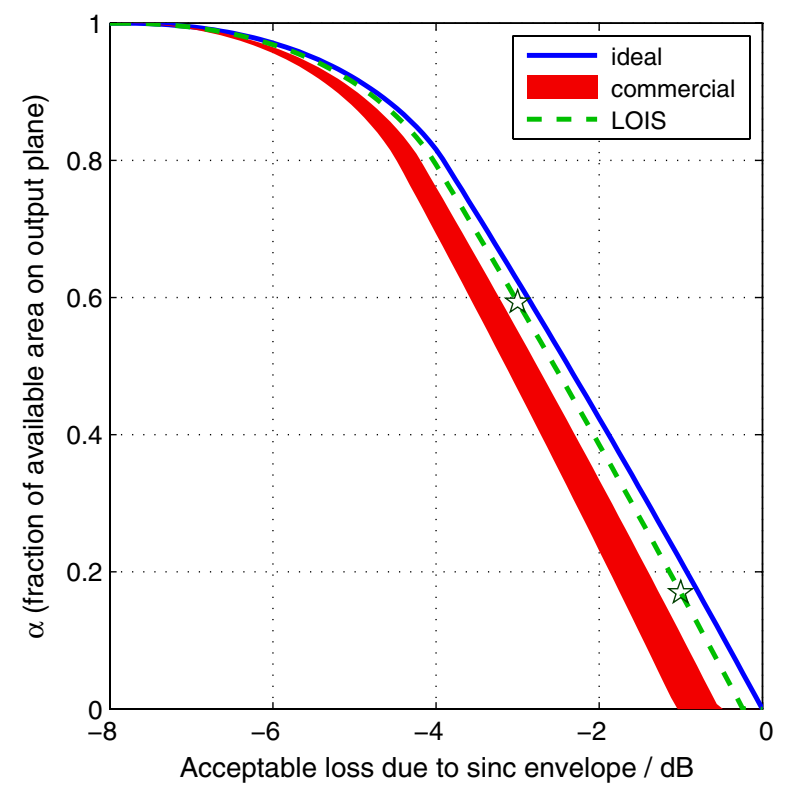

Fig. 10. (Color online) Acceptable loss due to the sinc envelope as a function of useful area fraction. The two stars correspond to the proposed points of operation for the LOIS device. 


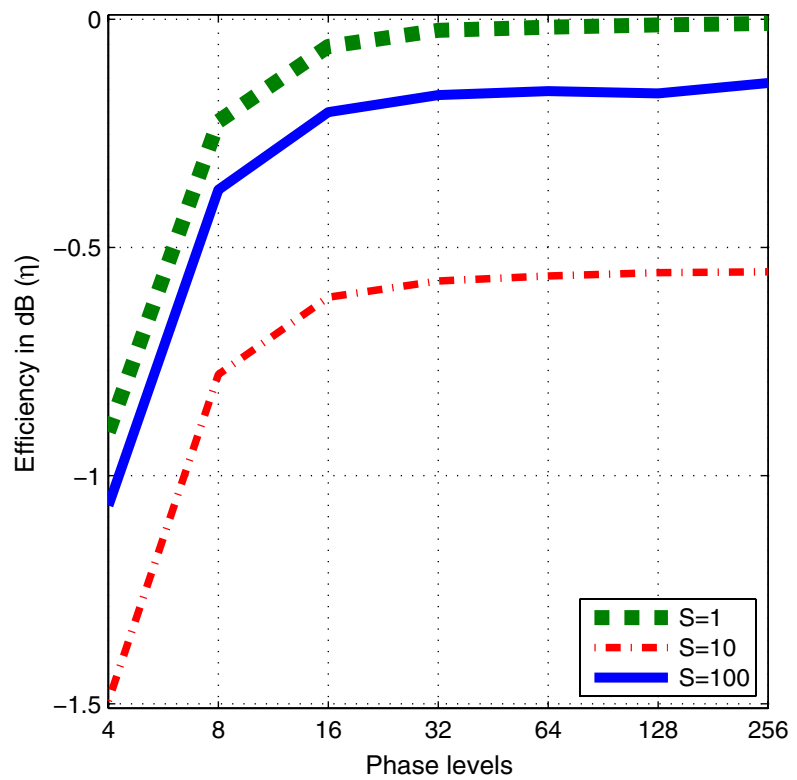

Fig. 11. (Color online) Diffraction efficiency of a phase-quantized hologram. From the top, efficiency for a single spot, a 100 spot, and a 10 spot generating hologram.

different quantization levels and then comparing their performance. This is shown in Fig. 11 for holograms with one, 10, and 100 output ports. Each efficiency point was calculated from 15 different holograms with $1024 \times 1024$ pixels. Loss due to phase quantization is negligible above 32 phase levels. It is therefore suggested to use at most 32 phase levels or 5 bits per pixel.

\section{Fringing Fields and Liquid Crystal Deformation}

The phase modulation in an LCOS device occurs in the liquid crystal material that is being rotated to the desired orientation by the electric field. Figure 12 shows a cross section of the liquid crystal layer on an LCOS device. As the thickness of the liquid crystal cell increases, the electric field between the pixels increases compared to the field between the pixel and the top electrode. This creates a smoothing effect for the phase profile that is affecting large phase transitions on the hologram, especially the $2 \pi$ phase jumps of a blazed grating. This $2 \pi$ phase jump is usually referred to as fly back.

Calculating the liquid crystal behavior on a large hologram with about a million pixels is computationally impossible. It requires the solution of the continuum theory equations for the entire device. There are a few computationally efficient approximations to estimate the behavior of the liquid crystal. In this paper, we use the low-pass filter approach, in which the phase profile is estimated by convolving the ideal profile with a kernel. The shape and width of the kernel is found by solving the continuum theory equations for two neighboring pixels using a finite element method (FEM) software [20].

The effect of electric fringing field, and thus the width of the kernel, increases as the thickness of the cell. So it is important to have as thin a cell as possible but that still achieves $2 \pi$ phase modulation. In practice, larger phase excursion is used to reduce the maximum rotation angle of the liquid crystal and increase speed. The thickness of the cell for a $\phi$ maximum phase excursion is given by

$$
d=\frac{1}{2} \frac{\phi}{2 \pi} \frac{\lambda}{\Delta n},
$$

where $\Delta n$ is the birefringence of the liquid crystal. The term $1 / 2$ arises because the device operates in reflection; thus, the wave is modulated in the way in and in the way out. For a $2.5 \pi$ phase modulation, $1.55 \mu \mathrm{m}$ wavelength, and using E7 liquid crystal, the cell thickness is $4.8 \mu \mathrm{m}$. Simulating this device using continuum theory in an FEM software gives the kernel of the low-pass filter. The kernel was found as described in [19].

Using this one-dimensional kernel, the efficiency of a blazed grating with all possible deflection angles was found and it is shown in Fig. 13. Fringing fields affect the diffraction efficiency of the grating, but the difference between the ideal sinc envelope efficiency (dotted curve) is small and never more than $-0.5 \mathrm{~dB}$. If both dimensions are considered, the maximum loss will be $-1 \mathrm{~dB}$. The maximum discrepancy between the two curves occurs when the period is roughly four pixels, i.e., the normalized deflection angle is 0.25 .

Two factors make Fig. 13 show the worst case scenario. First, in a blazed grating, the entire area of all the pixels contributes to the output port, and, thus, efficiency is unity. Any discrepancy will certainly create a profile with lower efficiency. For any other hologram, each pixel contributes to many output ports and a discrepancy in its phase will have a smaller effect into the output (this was verified by adding random noise to a blazed grating and a multicasting hologram and the effect on the latter was less). Second, part of the fringing fields occurs on the pixel boundary, were some of the loss has already been accounted for by the interpixel gap. As the
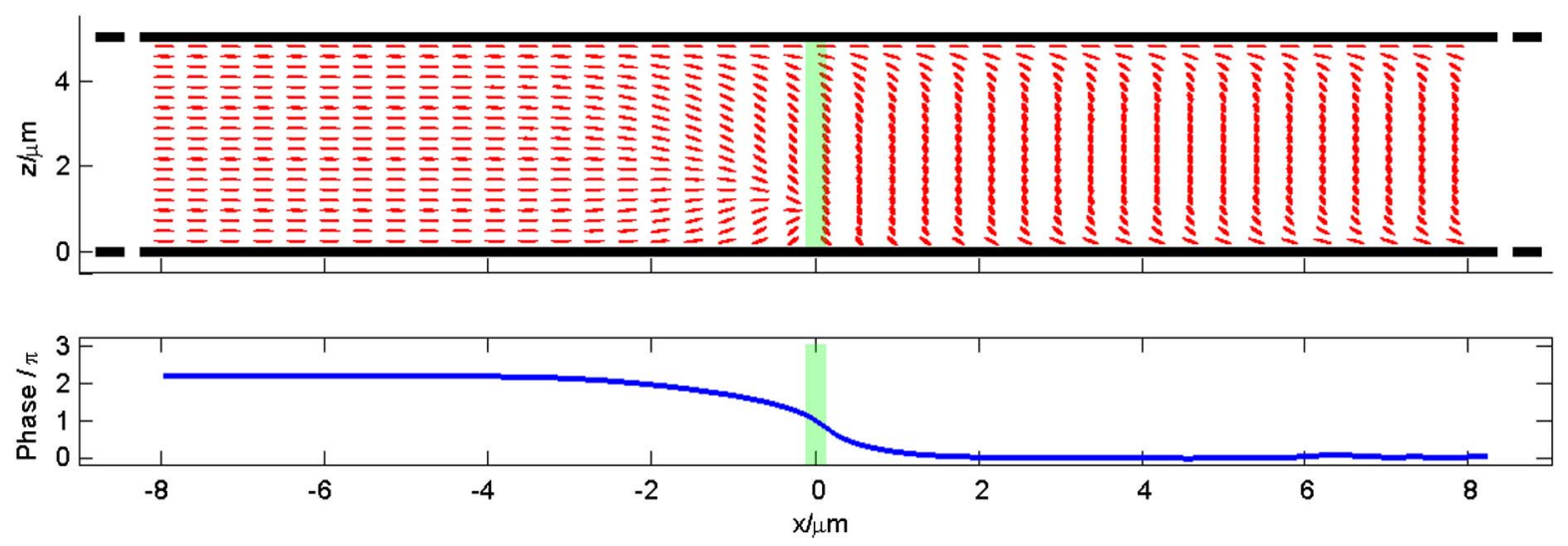

Fig. 12. (Color online) Fringing fields in an LCOS device between pixels smooths the phase transitions. 
size of the interpixel gap is smaller than the wavelength and the propagation distance within the liquid crystal considerably more, the effect of the interpixel gap cannot be easily accounted for (it can be accounted for by solving the Maxwell equations within the anisotropic liquid crystal and the metal electrodes [21]).

Fringing fields do change the shape of the sinc envelope on the output plane, as can be seen from Fig. 13. Consequently, so does the variation of $\alpha$ as a function of loss. This change, which is rather small, is accounted for together with the sinc envelope in Section 5 .

\section{OUTPUT PORTS}

The number of selected output fibers in a holographic interconnect is restricted by the diameter of the fibers and the accessible area on the output plane. The positioning of the output spots can be done with high accuracy by the hologram, but the point spread function of the spot, i.e., size of the spot, must be the same or smaller than the core of the output fibers $\left(d_{\mathrm{Fo}}\right)$. The area occupied by the cladding of the output fiber, with diameter $D_{\mathrm{Fo}}$, takes useful space but no output ports can be placed there. Assuming a rectangular grid, each output port will occupy $D_{\mathrm{Fo}} \times D_{\mathrm{Fo}}$ area on the output plane (see Fig. 3 ).

The area of the entire output plane is given by the maximum deflection of the hologram, which is

$$
u_{\max }= \pm \frac{\lambda f}{2 \Delta} \quad \text { or } \quad u_{\max }= \pm 0.5\left(N \frac{\lambda f}{L}\right)
$$

giving far-field area of $(N \lambda f / L)^{2}$. However, the useful area of the output plane is less because parts of the far field are highly attenuated by the sinc envelope. If only a fraction $\alpha$ of the farfield area is used, then efficiency is increased because the high-attenuation area is not used. This gives a total number of output fibers, $S_{\max }$, equal to

$$
S_{\max }=\alpha\left(\frac{N(\lambda f)}{L D_{\mathrm{Fo}}}\right)^{2}
$$

The wavelength and the focal distance terms may be eliminated by considering that the size of the output spot, $2 w_{o}$, must be smaller or equal to the output fiber core size, $d_{\mathrm{Fo}}$,

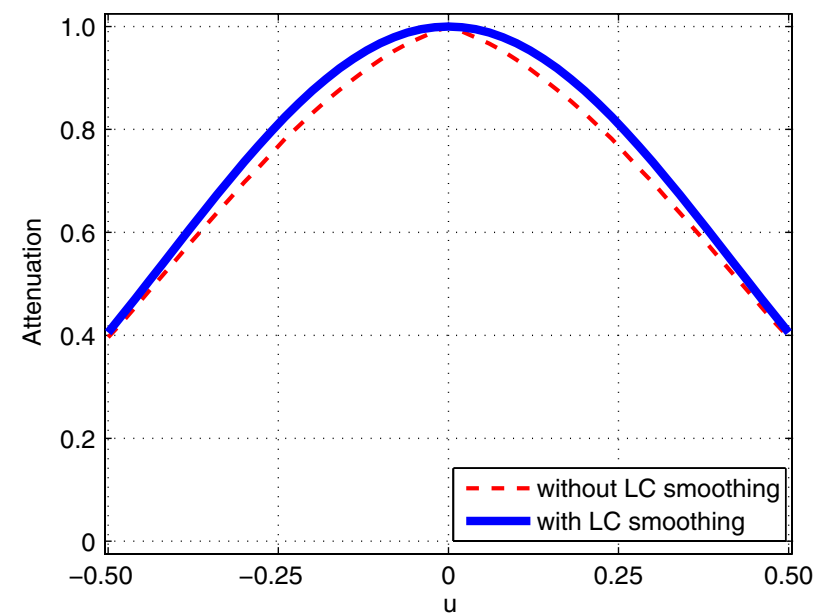

Fig. 13. (Color online) Diffraction efficiency of a blazed grating with and without fringing fields for a device with the characteristics of LOIS. such that

$$
2 w_{o}=\frac{4 \lambda}{\pi} \frac{f}{2 w_{d}} \leq d_{\mathrm{Fo}} \Rightarrow \lambda f \leq \frac{\pi}{4} 2 w_{d} d_{\mathrm{Fo}},
$$

giving the maximum number of output ports to be

$$
S_{\max }=\alpha\left(\frac{\pi}{4}\right)^{2}\left(\frac{d_{\mathrm{Fo}}}{D_{\mathrm{Fo}}}\right)^{2}\left(\frac{2 w_{d}}{L}\right)^{2} N^{2}
$$

The above equation contains five factors. The first factor, $\alpha$, states that the number of output ports, $S_{\max }$, can increase by reducing the efficiency of the system. The second factor, $\frac{\pi}{4}$, is determined by the packing factor of the fibers and it is equal to the ratio of the fiber's cross-section area over the area of a circumscribed square (for hexagonal packing this ratio will be $\frac{\pi}{2 \sqrt{3}}$, an increase of $\left.15 \%\right)$. The third term is determined by the relative size of the core to the cladding. For an $\mathrm{SMF}$, this is about $\frac{10 \mu \mathrm{m}}{125 \mu \mathrm{m}}$. The fourth term is determined by the apodization. Assuming the optimum apodization is used, it will be equal to 0.4 . Finally, the last term $N^{2}$, is the total number of pixels on the device.

It is interesting to note that the number of output ports is not a function of the wavelength or the focal length (though $w_{d}$ is determined by $f$ ). The number of output ports can also be increased by any amount by increasing the number of pixels, but it must also be accompanied by a suitable increase of the LCOS active area, $L \times L$, to keep $\alpha$ and, thus, the losses, constant.

\section{RESULTS AND DISCUSSION}

In the previous sections, the parameters affecting the performance of a holographic interconnect were determined. In this section, the system is considered as a whole and its characteristics are discussed with relation to different applications.

\section{A. Area}

The active area of the device is possibly the most important parameter of the system as it directly affects number of pixels, number of output ports, and cost. The cost of a device is directly linked to its area, with large devices being disproportionately expensive. As a guide, commercial silicon chips must be sufficiently small to keep the throughput high. The Intel Xeon x7460 processor, for example, has $503 \mathrm{~mm}^{2}$ die area and the Intel Xeon x 5405 has $214 \mathrm{~mm}^{2}$ area [22]. The JVC $4 \mathrm{k}$ LCOS device has active area of $546 \mathrm{~mm}^{2}$. The proposed devices have active areas ranging from 340 to $9 \mathrm{~mm}^{2}$. Even the top range LOIS device, with $340 \mathrm{~mm}^{2}$ area, is within the limits of current fabrication techniques, in terms of silicon area and cost. Note that the LCOS device in an optical interconnect would be only a small fraction of the cost. Installation, infrastructure, and other equipment will dominate the costs. This is unlike computers and projectors, where the product price is dominated by the cost of the CPU and the LCD, respectively.

\section{B. Number of Pixels}

If a small LCOS device is required, the area can be halved to $13.2 \mathrm{~mm} \times 13.2 \mathrm{~mm}$. This device ( $\mathrm{mLOIS}$ ) would have the same loss by keeping pixel size and pixel pitch the same but using half the output ports because of the decreased 
number of pixels. If even smaller active area is desired, a $9.1 \mathrm{~mm} \times 9.1 \mathrm{~mm}$ active area would introduce the same losses, but with a quarter of the users. The smallest device to consider has a $2.9 \mathrm{~mm} \times 2.9 \mathrm{~mm}$ active area. It would still have an acceptable number of users and with low cost. Below this size, there will be no substantial cost benefit and the optical design would become complex due to the small size.

\section{Output Ports}

When SMFs are used for output ports, their outer diameter is $D_{\mathrm{Fo}}=125 \mu \mathrm{m}$ and the core diameter is $d_{\mathrm{Fo}}=10 \mu \mathrm{m}$ (see Fig. 3). The beam width on the device is such that $2 w_{d} / L=$ 0.4 . For the proposed LOIS chip, $N=1024$ and $L=18.4 \mathrm{~mm}$. The value of $\alpha$ is chosen so that the desired trade-off between efficiency and the number of output ports is achieved. For $\alpha=0.17$, there are 112 output ports available, while, with $\alpha=0.59$, there are 390 output ports. For Fig. $\underline{3}$, where there are 192 ports, $\alpha=0.30$.

The number of ports may be increased or the active area of the LCOS device may be decreased by using an MMF, a taper fiber, or a microlens in front of an SMF instead of just an SMF. For a short span network, like a small neighborhood or a large building, where the fibers are only a few hundred meters long, MMF may provide the required bandwidth but with lower cost. When using an MMF, the core is substantially bigger compared with an SMF, 62.5 instead of $10 \mu \mathrm{m}$. However, the input fiber will remain an SMF with a small core. For the beam of the SMF to match the MMF core, the output plane must be optically magnified, while the spacing of the fibers will remain the same. The result is that more MMF fibers can be placed on the output plane or a smaller device may be used with fewer pixels. If the ratio $\left(\frac{d_{\mathrm{Fo}}}{D_{\mathrm{Fo}}}\right)$ increases by a factor of $\times 6.25$, a similar decrease can occur at $N$. Therefore, the number of pixels can be decreased from 1024 to 164 pixels with an active area of $2.9 \mathrm{~mm} \times 2.9 \mathrm{~mm}$ and the capabilities of the system in terms of port count and loss will remain as in Table $\underline{2}$.

\section{Efficiency}

Some of the system losses are affected by the number of output ports and some not. All the factors affecting system efficiency are shown in Table 2 . Apodization and fill factor incur the same losses to the interconnect irrespective of the number of output ports (see Subsections 3.A and 3.B), unlike the hologram efficiency (Subsection 3.C and Fig. 7), which is related to the number of output ports. The number of output ports, $S$, is not known, so the worst-case scenario is considered, which is $-0.9 \mathrm{~dB}$. The phase quantization when 5 bits per pixel are used is negligible and can be easily ignored (see Fig. 11). Finally, the sinc envelope attenuation and the fringing field losses must be considered together. The worst-case scenario is when the beam is deflected for a maximum angle. For $\alpha=0.59$, this attenuation is $-3.2 \mathrm{~dB}$ while, for $\alpha=0.17$, the attenuation is $-1.1 \mathrm{~dB}$. The mean value of loss, which could be more appropriate, was also calculated and shown in Table 2. Note that high loss fibers (e.g., long distance between interconnect and subscriber) should be placed closer to the center of the output plane and low loss links on the outer regions. Adding all the losses together, the overall mean efficiency of the system is $-1.8 \mathrm{~dB}$ if there are 112 ports and $-2.8 \mathrm{~dB}$ if there are 390 ports. Loss could also be seen as a
Table 2. Total Losses for the LOIS Device Taking

Two Scenarios: When Total Loss Is $-5.2 \mathrm{~dB}(\alpha=0.59)$ and When Total Loss Is $-3.2 \mathrm{~dB}(\alpha=0.17)$

\begin{tabular}{lcc}
\hline & \multicolumn{2}{c}{ Loss } \\
\cline { 2 - 3 } & $\begin{array}{c}\alpha=0.59 \\
390 \text { ports }\end{array}$ & $\begin{array}{c}\alpha=0.17 \\
\end{array}$ \\
\hline Apodization & $-0.1 \mathrm{~dB}$ & $-0.1 \mathrm{~dB}$ \\
Fill Factor & $-0.2 \mathrm{~dB}$ & $-0.2 \mathrm{~dB}$ \\
Hologram & $-0.9 \mathrm{~dB}$ & $-0.9 \mathrm{~dB}$ \\
Phase quantization & $-0.0 \mathrm{~dB}$ & $-0.0 \mathrm{~dB}$ \\
Sinc envelope + Fringing fields & $-1.6 \mathrm{~dB}$ & $-0.6 \mathrm{~dB}$ \\
& $(\max -3.2 \mathrm{~dB})$ & $(\max -1.1 \mathrm{~dB})$ \\
\hline Total loss & $-2.8 \mathrm{~dB}$ & $-1.8 \mathrm{~dB}$ \\
& $(\max -4.4 \mathrm{~dB})$ & $(\max -2.3 \mathrm{~dB})$ \\
\hline
\end{tabular}

reduction in the number of users. If the laser source of the system had just enough power for all the output ports, by reducing efficiency, the number of users also decreases.

\section{E. Proposed System}

The final system will depend on the needs of the network. Number of maximum users, $S_{\max }$, cost, and power available (and thus efficiency) are the three parameters that will determine the characteristics of the LCOS chip. Once the number of users is decided, the relationship between loss $(\alpha)$ and the number of pixels $(N)$ is determined. Increasing the number of pixels while keeping the number of users the same will increase the active area $L \times L$ and, thus, the cost of the device. At the same time, $\alpha$ decreases and so do the losses.

The first proposed system will use the LOIS device. Such a device could be used for the backbone of an HDTV distribution system. The input to the switch will be an SMF connected to a laser. It will require less than 100 ports and low losses. Possibly, at any point, only 10 to 20 outputs are connected, thus the power at the output fibers remains high but the system retains the capability to shift the power to any output in case of a link failure.

The second proposed system will also have as input an SMF connected to a laser. However, this system will be used within a neighborhood and the output ports will have larger diameter (MMF, taper fiber, or an SMF with a microlens). A $850 \mathrm{~nm}$ laser can be used too. The number of users per neighborhood will be more than 100 and all of them may be connected. The cost of the device will be a more important factor as more of these devices will be deployed so smaller LCOS devices will be used. The $n$ LOIS device can be used with loss of -2.8 to $-4.4 \mathrm{~dB}$ and with about 390 ports ( $\alpha=0.59$ in Table 2 ).

For the third system, a VCSEL may be used as an input to the interconnect. As VCSELs have larger beam width than a laser, the required magnification will be less than $\times 6.25$ when used with MMF. This effectively reduces the number of ports. Devices like the $m$ LOIS and $\mu$ LOIS may be used in the cases when the output ports are MMF and the input is a VCSEL. Also, if the fiber grid has a spacing of 250 instead of $125 \mu \mathrm{m}$, the number of output ports will also decrease by a factor of 4 . Fiber ribbons with $250 \mu \mathrm{m}$ spacing are widely available. Again, the $m$ LOIS and $\mu$ LOIS devices with more pixels can provide the necessary port count for a switch that uses MMF for outputs. 


\section{CONCLUSIONS}

In this work, we investigated the use of LCOS devices for multicasting optical interconnects. Mathematical formulas to link the characteristics of the system-efficiency, number of output ports, pixel number, and device area-were presented. This enables the optical engineer to best use the resources of the device. A number of devices were presented and it was found that even very small devices with active area of only $9 \mathrm{~mm}^{2}$ can be used for multicasting optical signals. Larger devices with areas of up to $339 \mathrm{~mm}^{2}$ can be used for distributing signals to SMF with very low loss. The number of output ports and, thus, subscribers is large with more than hundreds of ports per switch. It can be further increased by enlarging the diameter of the output ports.

Concluding, LCOS devices could be the way to multicast optical signals to the house in the near future. Their cost, flexibility, reliability, and large number of output ports make them the ideal solution. As the power of lasers increases and, similarly, the bandwidth requirements increase, it becomes clear that performing the signal distribution in the optical domain is essential and LCOS devices can do that in a very effective way.

\section{ACKNOWLEDGMENTS}

Jeroen Beeckman is Postdoctoral Fellow of the Research Foundation Flanders (FWO).

\section{REFERENCES}

1. M. Komarcevic, I. G. Manolis, T. D. Wilkinson, and W. A. Crossland, "Polarization effects in reconfigurable liquid crystal phase holograms," Opt. Commun. 244, 105-110 (2005).

2. K. L. Tan, S. T. Warr, M. G. Ilias, T. D. Wilkinson, M. M. Redmond, A. W. Crossland, and B. Robertson, "Dynamic holography for optical interconnections. I. Noise floor of lowcrosstalk holographic switches,” J. Opt. Soc. Am. A 18, 195-204 (2001).

3. K. L. Tan, S. T. Warr, M. G. Ilias, T. D. Wilkinson, M. M. Redmond, A. W. Crossland, and B. Robertson, "Dynamic holography for optical interconnections. II. Routing holograms with predictable location and intensity of each diffraction order,” J. Opt. Soc. Am. A 18, 205-215 (2001).

4. C. A. T. H. Tee, W. A. Crossland, T. D. Wilkinson, and A. B. Davey, "Binary phase modulation using electrically addressed transmissive and silicon backplane spatial light modulators," Opt. Eng. 39, 2527-2534 (2000).

5. P. Evans, G. Baxter, H. Zhou, D. Abakoumov, S. Poole, and S. Frisken, "LCOS-based WSS with true integrated channel monitor for signal quality monitoring applications in ROADMS," in Optical Fiber Communication Conference and Exposition and The National Fiber Optic Engineers Conference (Digest) (Optical Society of America, 2008), pp. 1-3.
6. W. A. Crossland, I. G. Manolis, M. M. Redmond, K. L. Tan, T. D. Wilkinson, M. J. Holmes, T. R. Parker, H. H. Chu, J. Croucher, V. A. Handerek, S. T. Warr, B. Robertson, I. G. Bonas, R. Franklin, C. Stace, H. J. White, R. A. Woolley, and G. Henshall, "Holographic optical switching: the ROSES demonstrator," J. Lightwave Technol. 18, 1845-1854 (2000).

7. P. Chanclou, H. Ramanitra, P. Gravey, and M. Thual, "Design and performance of expanded mode fiber using microoptics," J. Lightwave Technol. 20, 836-842 (2002).

8. X. Zheng, V. Kaman, S. Yuan, Y. Xu, O. Jerphagnon, A. Keating, R. C. Anderson, H. N. Poulsen, B. Liu, J. R. Sechrist, C. Pusarla, R. Helkey, D. J. Blumenthal, and J. E. Bowers, "Threedimensional MEMS photonic cross-connect switch design and performance," IEEE J. Sel. Top. Quantum Electron. 9, 571-578 (2003).

9. M. Anisetti, C. Ardagna, V. Bellandi, and E. Damiani, "Telecentric and achromatic $F$-theta scan lens system and method of use," U.S. patent 5,404,247 (4 April 1995).

10. T. E. Stern, G. Ellinas, and K. Bala, Multiwavelength Optical Networks: Architectures, Design, and Control (Cambridge University, 2009).

11. JVC, "JVC develops the worlds smallest 1.27 inch $4 \mathrm{~K} 2 \mathrm{~K}$ D-ILA device" (June 2007), www.jvc.co.jp.

12. A. Georgiou, T. D. Wilkinson, N. Collings, and W. A. Crossland, "Algorithm for computing spot-generating holograms," J. Opt. A Pure Appl. Opt. 10, 015306 (2008).

13. A. Kirk and T. Hall, "Design of binary computer generated holograms by simulated annealing: coding density and reconstruction error," Opt. Commun. 94, 491-496 (1992).

14. M. A. Seldowitz, J. P. Allebach, and D. W. Sweeney, "Synthesis of digital holograms by direct binary search," Appl. Opt. 26, 2788-2798 (1987).

15. A. Georgiou, M. Komarcevic, and W. A. Crossland, "Noise suppression in liquid crystal beam steering devices," presented at SPIE Great Lakes Photonics Symposium, 12-16 June, 2006, Dayton, Ohio, USA.

16. T. Cizmar, M. Mazilu, and K. Dholakia, "In situ wavefront correction and its application to micromanipulation," Nat. Photon. 4, 388-394 (2010).

17. E. Hecht, Optics, 4th ed. (Addison Wesley, 2002).

18. U. Efron, B. Apter, and E. Bahat-Treidel, "Fringing-field effect in liquid-crystal beam-steering devices an approximate analytical model," J. Opt. Soc. Am. A 21, 1996-2008 (2004).

19. A. G. Georgiou, M. Komarcevic, T. D. Wilkinson, and W. A. Crossland, "Hologram optimisation using liquid crystal modelling," Mol. Cryst. Liq. Cryst. 343, 511-526 (2005).

20. R. James, M. C. Gardner, F. A. Fernández, and S. E. Day, “3D modelling of high resolution devices," Mol. Cryst. Liq. Cryst. 450, 105-118 (2006).

21. P. Vanbrabant, J. Beeckman, K. Neyts, R. James, and F. A. Fernandez, "A finite element beam propagation method for simulation of liquid crystal devices," Opt. Express 17, 10895-10909 (2009).

22. Intel Corporation, Intel Corporation website (2010). http://ark. intel.com

23. Brillian, "Brillian 2-Megapixel BR1920HC," www.brilliancorp .com. 